\title{
Vegetation Database Forests of the Oldenburg Region
}

\author{
Cord Peppler-Lisbach
}

\begin{abstract}
The Vegetation Database Forests of the Oldenburg Region (GIVD ID EU-DE-010) contains numerous forest releves from the surrounding area of Oldenburg within a radius of ca. $50 \mathrm{~km}$. These relevés originate from several studies conducted by scientists and students of Oldenburg University since 1990. A high proportion of relevés includes information on $\mathrm{pH}$ and humus form. The relevés are mostly unpublished but the results have led to several publications especially on the role of $\mathrm{pH}$ in shaping floristic patterns of forest communities.
\end{abstract}

Keywords: deciduous forest; NW Germany; Oldenburg; Querco-Fagetea.

GIVD Database ID: EU-DE-010

Last update: $2012-07-08$

\section{Vegetation Database Forests of the Oldenburg Region}

Scope: woodland relevés from the Oldenburg region (Northwestern Germany)

mainly data from diploma theses, ca. $20 \%$ published
Status: ongoing capture
Period: $1990-2008$

Database manager(s): Cord Peppler-Lisbach (cord.peppler.lisbach@uni-oldenburg.de)

Owner: [NA]

Web address: [NA]

Availability: free upon request

Database format(s): TURBOVEG

Online upload: no

Online search: no

Publication: [NA]

Plot type(s): normal plots

Non-overlapping plots: 1,522

Total plot observations: 1,522

Export format(s): TURBOVEG, Excel, CSV file

Countries: DE: $100.0 \%$

Forest: $100 \%$ - Non-forest: aquatic: $0 \%$; semi-aquatic: $0 \%$; arctic-alpine: $0 \%$; natural: $0 \%$; semi-natural: $0 \%$; anthropogenic: $0 \%$

Guilds: all vascular plants: $100 \%$; bryophytes (terricolous or aquatic): $70 \%$

Environmental data: altitude: $60 \%$; surface cover other than plants (open soil, litter, bare rock etc.): $70 \%$; soil pH: $80 \%$

Performance measure(s): cover: $100 \%$

Geographic localisation: GPS coordinates (precision $25 \mathrm{~m}$ or less): $40 \%$; point coordinates less precise than GPS, up to $1 \mathrm{~km}$ : $40 \%$; small grid (not coarser than $10 \mathrm{~km}$ ): $20 \%$

Sampling periods: $1990-1999: 60.0 \%$; $2000-2009: 40.0 \%$

Information as of 2012-07-12; further details and future updates available from http://www.givd.info/ID/EU-DE-010

Cord Peppler-Lisbach (cord.peppler.lisbach@uni-oldenburg.de)

Institut für Biologie und Umweltwissenschaften, Universität Oldenburg,Postfach 2503, 26111 Oldenburg, GERMANY 